\title{
Buch oder Internet
}

\section{Erhard Taverna}

Dr. med., Mitglied der Redaktion

Viele Argumente werden gegen das gedruckte Buch vorgebracht. Erstens sei es zu schwerfällig, um dem heutigen Tempo der Wissensproduktion zu entsprechen, siehe das Verschwinden vieler renommierter Nachschlagewerke, wie Brockhaus oder Encyclopaedia Britannica. Zweitens sei das Buch individualistisch, elitär und hierarchisch, und drittens stehe es der gerechteren Wissensproduktion und Präsentation im Wege. In Horizonte [1], dem Forschungsmagazin des Schweizerischen Nationalfonds, werden, als ultimativer Fortschritt, 200 Jahre Weltliteratur in 0,4 Sekunden vorgestellt. Langsames Lesen sei out, heute würden Bücher im Nu mit dem Computer durchstöbert und erforscht. Literatur und Sprache mit Zahlen zu analysieren sei ein Markenzeichen exakter Wissenschaft. Netz-Esoteriker und Bibliophile unterscheiden zwei Lesertypen. Die einen sehen das Buch als Störfaktor. More scanning, less reading, das Überfliegen kurzer Texte spart Zeit. Vernetzen, Verlinken und Assoziieren ermöglicht eine neue Strategie des Denkens, ein multimediales Navigieren durch unterschiedlichste Wissensräume, ein Eldorado für Smartphones. Die anderen betonen die Vorzüge des Close oder Deep Reading, einer Kulturtechnik, die komplexe sensomotorische Fähigkeiten voraussetzt, die fokussiertes Lesen und verschiedenste Lektüreerfahrungen erst ermöglicht, sei es narrativ, analytisch, selektiv-gründlich oder auch querlesend, zufällig und assoziativ. Die Biomedizinische Informatik als eigenständige Disziplin ist nun endlich auch in der Schweiz angekommen, moniert das Bulletin der Akademie der Schweizerischen Wissenschaften SAMW [2].

Enorme und ständig grösser werdende Mengen an unterschiedlichsten Daten gelte es für die medizinische Forschung und Gesundheitsversorgung zu dokumentieren und auszubeuten. Als Beispiel für Algorithmen zur Entscheidungsfindung gilt ausgerechnet der hochgradig problematische, automatisierte Börsenhandel. Nicht zufällig erwähnen die Autoren quantitative Messverfahren wie Impact-Faktoren, die auf Zitationsanalysen beruhen. Die Messwerte entsprechen der Ökonomisierung akademischer Forschung. Sie werden von Medienkonzernen erstellt und ähnlich wie die Börsenkurse in Geld umgesetzt. Kurzfristig scheinen die Biomedical Informatics vor allem die Administration zu fördern. Planung, Koordination von Prozessen, Ressourcenplanung, Effizienzkontrollen, Qualitätssicherung, Auditing und kompetitive Dienstleistungen erfüllen die Auflagen der neoliberalen Marktvorgaben. Noch ist die Illusion der Datensicherheit kein Thema.
Wissen als Ware ist Ausdruck der gleichen Ideologie. Turn your idea into business war einmal das Logo über dem Hauptportal der ETH Zürich. Startups sind keine schlechte Idee. Horizonte stellt die Frage, ob Universitäten, die Forschungsarbeiten mit öffentlichen Geldern ermöglicht haben, am kommerziellen Erfolg dieser Unternehmen teilhaben sollen oder nicht. Anzahl und Erfolg der Jungunternehmer beeinflussen das Ranking der Universität. Der Rang vergleicht weniger die Qualität als vielmehr die Anpassung an das hegemoniale angelsächsische Geschäftsmodell (Antonio Loprieno, ehemals Rektor der Uni Basel). Öffentlich gefördertes Wissen wird vorrangig als ökonomische Ressource betrachtet, die Wirtschaft und Wohlstand fördern soll. Wachstum und soziale Beschleunigung haben das Publikationswesen umgekrempelt. Beispielhaft dafür sind die Auswirkungen der Open-AccessInitiative 2002, die durch den Hedge-Fonds-Milliardär George Soros gefördert wurde. Alle wissenschaftlichen Arbeiten sind für Benutzer kostenlos ins Netz zu stellen. Wissen soll Gemeinbesitz und nicht Privateigentum sein. Cyberspace wird zur Heimat des neuen, demokratischen Geistes, die müden Giganten aus Papier und Leim haben ausgedient, die neue Aufklärung kann auf sie verzichten. Was aus den guten Absichten geworden ist, wo sich die vielen Akteure heute befinden, beschreibt der ETH-Professor für Wissenschaftsforschung Michael Hagner in seinem Buch Zur Sache des Buches [3].

Verlage, IT-Unternehmen, Startups, Beraterfirmen, Bibliotheken, Politiker, Bürokraten und Netzaktivisten haben im Open-Access-Modell inzwischen eine Lizenz zum Gelddrucken entdeckt. Geisteswissenschaftliche Bücher, so der Autor, stehen der neoliberalen Impact-Rhetorik im Wege. Obwohl oder gerade weil ihr Wirkungszyklus wesentlich länger dauert als der von naturwissenschaftlichen Artikeln mit einer kurzen Halbwertszeit, die kaum zehn Jahre im Zitationskarussel überdauern. Bücher überleben auch physisch länger, denn digitale Speicherformen zerfallen schnell zum digitalen Schutt, wie auch Horizonte weiss. Laut Bundesarchiv in Bern ist die digitale Konservierung eines Meters Archivmaterial neunmal teurer als dessen analoge Konservierung. Das notwendige Kopieren auf neue Formate wird eine aufwendige und kostspielige Daueraufgabe sein.

Es gehe darum, das Netz zu nutzen, um bessere Texte für das Papier zu schreiben, so der Autor. Denn ein Stück von jener Intelligibilität der Welt werde nur ein Buch zum Vorschein bringen können. 